\title{
Review of levetiracetam, with a focus on the extended release formulation, as adjuvant therapy in controlling partial-onset seizures
}

This article was published in the following Dove Press journal:

Neuropsychiatric Disease and Treatment

9 September 2009

Number of times this article has been viewed

\author{
Carol M Ulloa \\ Allen Towfigh \\ Joseph Safdieh \\ Department of Neurology \\ and Neuroscience, Weill Medical \\ College of Cornell University, \\ New York, NY, USA
}

Correspondence: Joseph Safdieh Department of Neurology and Neuroscience, Weill Medical College of Cornell University, 520 East 70th Street

Starr 607

New York, NY 10021

Email jos9046@med.cornell.edu

\begin{abstract}
Levetiracetam is a second-generation antiepileptic drug (AED) with a unique chemical structure and mechanism of action. The extended release formulation of levetiracetam (Keppra XR ${ }^{\mathrm{TM}}$; UCB Pharma) was recently approved by the Food and Drug Administration for adjunctive therapy in the treatment of partial-onset seizures in patients 16 years of age and older with epilepsy. This approval is based on a double-blind, randomized, placebo-controlled, multicenter, multinational trial. Levetiracetam XR allows for once-daily dosing, which may increase compliance and, given the relatively constant plasma concentrations, may minimize concentration-related adverse effects. Levetiracetam's mode of action is not fully elucidated, but it has been found to target high-voltage, N-type calcium channels as well as the synaptic vesicle protein 2A (SV2A). Levetiracetam has nearly ideal pharmacokinetics. It is rapidly and almost completely absorbed after oral ingestion, is $<10 \%$ protein-bound, demonstrates linear kinetics, is minimally metabolized through a pathway independent of the cytochrome P450 system, has no significant drug-drug interactions, and has a wide therapeutic index. The most common reported adverse events with levetiracetam XR were somnolence, irritability, dizziness, nausea, influenza, and nasopharyngitis. Levetiracetam XR provides an efficacious and well-tolerated treatment option for adjunctive therapy in the treatment of partial-onset seizures.
\end{abstract}

Keywords: levetiracetam, partial-onset seizures, antiepileptic drugs

\section{Introduction}

Extended release levetiracetam (Keppra $\mathrm{XR}^{\mathrm{TM}}$; UCB Pharma) is a relatively new antiepileptic drug (AED) with a unique chemical structure and mechanism of action. Its use is indicated by the Food and Drug Administration (FDA) for adjunctive therapy in the treatment of partial-onset seizures in patients 16 years of age and older. This approval is based on a recent double-blind, randomized, placebo-controlled, multicenter, multinational trial that included 158 patients between the ages of 12 and $70 .{ }^{1}$

Levetiracetam was originally approved by the US FDA and the European Medicines Evaluation Agency in 1999 as adjunctive treatment for partial-onset seizures in adults. This approval was based on the results of 3 double-blind, randomized, multicenter trials including a total of 904 patients..$^{2-4}$

Levetiracetam XR was developed to provide patients with the convenience of once-daily dosing, which may improve drug compliance and tolerability. ${ }^{1,5}$

\section{Mechanism of action}

While much progress has been made to elucidate the mode of action of levetiracetam, its precise mechanism of action is still not fully understood. The mechanism of action 
of levetiracetam is distinct from first-generation and other second-generation AEDs. Levetiracetam does not share a high affinity for many known targets for existing AEDs including phenytoin, carbamazepine, sodium valproate, phenobarbital, dimethadione or benzodiazepines. ${ }^{6}$ In addition, levetiracetam appears not to work by the three classic routes of other AEDs: sodium channel modulation, low-voltage-activated (T-type) calcium channel modulation, and direct gamma-aminobutyric acid (GABA) facilitation.

\section{Animal models}

Levetiracetam is devoid of anticonvulsant activity in the two classic acute seizure models used for AED screening, including the maximal electric shock seizure test and the pentylenetetrazol seizure test. However, it does demonstrate anticonvulsive effects in the acute corneal electroshock model and the selective chemoconvulsant seizure models, including pilocarpine and kainic acid induced models. ${ }^{7}$

Kindling, the phenomenon of repeated application of an initially subconvulsive stimulus leading to development of spontaneous seizures of increasing severity and duration, is a reliable way to examine the anticonvulsive and antiepileptogenic properties of AEDs. ${ }^{8}$ The rate of kindling progression is used as a marker of antiepileptogenic potency. Levetiracetam exerts a significant antiepileptic effect, even after discontinuation of therapy, in kindled models and in the double mutant (tm/tm, zi/zi) spontaneously epileptic rat (SER) model, which exhibits both tonic and absence-like seizures. ${ }^{7,9-11}$

\section{Ion channels}

Levetiracetam has no inhibitory effect on sodium or low-voltage-activated, T-type calcium channels in rat neocortical neurons ${ }^{12}$ and does not have a high affinity for GABA, N-methyl d-aspartate (NMDA) or glutamate receptors. . $^{6,11}$ Levetiracetam reduces high-voltage, N-type, calcium channel currents in isolated rat CA1 hippocampal neurons but does not have an effect on L-type, P-type or Q-type calcium channels. $^{13,14}$ This effect occurs within a few seconds and suggests a direct interaction of levetiracetam with the N-type channel, rather than a second messenger pathway.

Levetiracetam has also been shown to reduce caffeine induced calcium currents in rat hippocampal cell cultures, ${ }^{15}$ which may facilitate GABA transmission indirectly. ${ }^{16,17}$ In addition, levetiracetam has been demonstrated to antagonize neuronal hypersynchronization in rat hippocampal slices in high-potassium, low-calcium bathing fluid. ${ }^{18}$

\section{Synaptic vesicle protein $2 \mathrm{~A}$}

Early work by Noyer et al suggested a protein-binding site that was highly specific to central nervous system (CNS) synaptic plasma membranes. ${ }^{6}$ More recently, that site was identified by Lynch et al as the synaptic vesicle protein $2 \mathrm{~A}$ (SV2A), a plasma membrane protein of approximately $90 \mathrm{kDa}$ that is nearly ubiquitous throughout the CNS. ${ }^{19}$ Levetiracetam binds reversibly, saturably, and stereo-specifically to this receptor site. ${ }^{6,15,19,20}$

The exact role of the SV2A protein is not fully understood; it is thought to participate in the physiologic functioning of the synaptic vesicle. ${ }^{19}$ Its role in epilepsy is supported by the fact that SV2A knock-out mice develop seizures soon after birth and usually die within 3 weeks. ${ }^{21}$ Furthermore, the affinity of various compounds to the SV2A binding site correlates strongly with their anti-seizure potency in the audiogenic epilepsy mouse model. ${ }^{19}$

\section{Pharmacokinetic profile Physical and chemical properties}

Keppra XR tablets are film-coated extended release tablets that contain $500 \mathrm{mg}$ of levetiracetam, a structurally unique AED. Levetiracetam is the S-enantiomer of $\alpha$-ethyl-2-oxo1-pyrrolidine acetamide. Its molecular formula is $\mathrm{C}_{8} \mathrm{H}_{14} \mathrm{~N}_{2} \mathrm{O}_{2}$, and its molecular weight is 170.21. Levetiracetam is a white to off-white crystalline powder that has a faint odor and bitter taste. $^{22}$ It is essentially water soluble.

\section{Absorption and distribution}

Levetiracetam is almost completely absorbed after oral administration, exhibits $>95 \%$ bioavailability, has a volume of distribution of 0.5 to $0.7 \mathrm{~L} / \mathrm{kg}$ (close to that of intracellular and extracellular water), and in rats has been shown to rapidly penetrate the brain without brain region specificity. In addition, levetiracetam is not significantly protein bound $(<10 \%)$. $^{22,23}$

In a recent study by Rouits et al $1000 \mathrm{mg}$ of levetiracetam XR (two $500 \mathrm{mg}$ XR tablets) was found to be bioequivalent to two $500 \mathrm{mg}$ immediate release (IR) tablets given 12 hours apart, with comparable maximal plasma concentrations $\left(\mathrm{C}_{\max }\right)$ and area under the plasma concentration vs time (AUC). Under fasting conditions, the median time to maximal plasma concentration $\left(\mathrm{T}_{\max }\right)$ was 4 hours for levetiracetam XR vs 0.9 hours for levetiracetam IR (Table 1). Time to peak was further prolonged by 2.5 hours when the XR tablet was given after intake of a high-fat, high-calorie breakfast. However, the AUC and $\mathrm{C}_{\max }$ were similar to those in the fasting state. Furthermore, based on the slopes of the regression vs dose, 
Table I Bioequivalence of levetiracetam IR and XR under fasting conditions

\begin{tabular}{lll}
\hline Parameter & $\begin{array}{l}\text { Levetiracetam } \\
\text { IR 500 } \mathbf{~ m g ~ q ~} \mathbf{2} \mathbf{~ h}\end{array}$ & $\begin{array}{l}\text { Levetiracetam } \\
\text { XR } \mathbf{0 0 0} \mathbf{~ m g}\end{array}$ \\
\hline $\mathrm{C}_{\text {max }}(\mu \mathrm{g} / \mathrm{mL})$ & 19.7 & 17.4 \\
$\mathrm{~T}_{\text {max }}(\mathrm{h})$ & 0.9 & 4 \\
$\mathrm{AUC}_{(0-\mathrm{t})}(\mu \mathrm{g} / \mathrm{mL})$ & 317 & 307 \\
$\mathrm{AUC}_{\infty}((\mu \mathrm{g} / \mathrm{mL})$ & 325 & 313 \\
\hline
\end{tabular}

Notes: $\mathrm{AUC}_{(0-t)}$ : area under the plasma-concentration-time curve from time 0 to last measurable time point.

$\mathrm{AUC}_{\infty}$ : area under the plasma-concentration-time curve extrapolated from time 0 to infinity.

Adapted with permission from Rouits E, Burton I, Guénolé E, Troenaru MM, Stockis A, Sargentini-Maier ML. Pharmacokinetics of levetiracetam XR $500 \mathrm{mg}$ tablets. Epilepsy Res. 2009;84(2-3):224-231..$^{5}$ Copyright (C) 2009 Elsevier.

the $\mathrm{C}_{\max }$ and $\mathrm{AUC}$ were dose proportional after a single dose of $1000 \mathrm{mg}, 2000 \mathrm{mg}$, and $3000 \mathrm{mg}$ levetiracetam XR. The half-life, apparent clearance, and apparent volume of distribution were dose-independent. Therefore, this study concluded that: (1) levetiracetam XR $1000 \mathrm{mg}$ once a day is bioequivalent to levetiracetam IR $500 \mathrm{mg}$ bid; (2) there is no food effect; and (3) the absorption of levetiracetam XR is dose proportional from $1000 \mathrm{mg}$ to $3000 \mathrm{mg} .{ }^{5}$

\section{Metabolism and elimination}

The metabolism of levetiracetam is independent of the hepatic cytochrome P450 system. Sixty-six percent of an administered dose of levetiracetam is excreted unchanged in the urine, and $27 \%$ is excreted as inactive metabolites. ${ }^{24}$ The major metabolic pathway involves enzymatic hydrolysis of the acetamide group, which results in one major inactive carboxylic acid metabolite (L057). ${ }^{23}$

Levetiracetam is rapidly cleared, with about $93 \%$ of a given dose excreted within 48 hours of oral administration. The rate of renal clearance is $0.6 \mathrm{~mL} / \mathrm{min} / \mathrm{kg}$. Levetiracetam's plasma half-life in healthy subjects is approximately 6 to 8 hours. ${ }^{23}$ Half-life and apparent clearance of both levetiracetam IR and XR are dose-independent. ${ }^{5}$ The mechanism of excretion is through glomerular filtration, followed by partial tubular reabsorption. The major metabolite, L057, is excreted by glomerular filtration and active tubular secretion with a renal clearance of $4 \mathrm{~mL} / \mathrm{min} / \mathrm{kg} .{ }^{23}$

The rate of elimination of levetiracetam is dependent on creatinine clearance; therefore, dose adjustments are required in patients with renal impairment (Table 2). ${ }^{22}$ The plasma elimination half-life of levetiracetam IR in elderly subjects increases to between 10 and 11 hours. ${ }^{24}$ This is likely due to an age-related decrease in creatinine clearance, which may require a decrease in daily dose.
The pharmacokinetics of levetiracetam IR were studied in 16 patients with varying degrees of liver cirrhosis. ${ }^{25}$ Patients with mild to moderate hepatic dysfunction require no adjustment of levetiracetam dose. However, those with severe cirrhosis and concomitant renal insufficiency (hepato-renal syndrome) may require a lower levetiracetam dose.

\section{Pharmacokinetics in pediatric patients}

The pharmacokinetic profile of levetiracetam XR has not been studied in patients below the age of 16 . However, the pharmacokinetics of a single $20 \mathrm{mg} / \mathrm{kg}$ dose of levetiracetam IR in 12 children aged 2 to 46 months was studied. ${ }^{26}$ The mean half-life was $5.3 \pm 1.3$ hours. This is slightly shorter than in children aged 6 to 12 years, previously reported at $6 \pm 1.1$ hours. ${ }^{27}$ In addition, the apparent clearance $(1.46 \pm 0.42 \mathrm{~mL} / \mathrm{min} / \mathrm{kg})$ was more rapid than that seen in adults.

\section{Pharmacokinetic interactions}

Levetiracetam has no known clinically significant drug interactions. In vitro studies have demonstrated that neither levetiracetam nor its major metabolite have an effect on various cytochrome P450 isoenzymes. ${ }^{23}$ Several studies in healthy subjects have demonstrated no pharmacokinetic interaction between levetiracetam and phenytoin, ${ }^{28}$ warfarin, ${ }^{29}$ digoxin, ${ }^{30}$ and an oral contraceptive containing ethinyl estradiol and levonorgestrel. ${ }^{31}$ In addition, a pooled analysis of data from several randomized clinical trials showed that adjunctive therapy with levetiracetam did not influence the steady-state concentrations of concomitantly administered carbamazepine, phenytoin, valproate, lamotrigine, gabapentin, phenobarbital or primidone. ${ }^{32}$

An earlier pooled analysis investigated the effects of various AEDs on the pharmacokinetics of levetiracetam..$^{33}$ This study showed that, although there was a trend towards lower serum levetiracetam levels and a shorter half-life in patients on concomitant enzyme-inducing AEDs, the estimated half-life of levetiracetam in these patients was still within the expected 6- to 8-hour range for healthy subjects and the differences in pharmacokinetic parameters were considered unlikely to be clinically significant.

\section{Clinical efficacy Pivotal trial for extended release levetiracetam}

In a recent double-blind, randomized, placebo-controlled, multicenter, multinational trial of levetiracetam $\mathrm{XR}$ as adjunctive treatment of partial-onset seizures with or without secondary generalization in adults with epilepsy, the drug's efficacy 
and tolerability were demonstrated. ${ }^{1}$ One hundred fifty-eight patients (intention-to-treat population) between 12 and 70 years of age with a diagnosis of partial-onset seizures refractory to 1 to 3 AEDs were randomized to levetiracetam XR $(n=79)$ or placebo $(n=79)$. The most frequently used concomitant AEDs were carbamazepine (53\%), valproate (37\%), topiramate (20\%), lamotrigine (19\%), phenytoin (13\%), clobazam $(10 \%)$ and phenobarbital $(9.5 \%)$. The trial began with an 8 -week baseline period in which eligibility was determined. Thereafter, patients were randomized to receive either levetiracetam XR 1000 mg daily or placebo during the 12-week treatment period. There was no uptitration period. The primary efficacy variable was based on the frequency of partial-onset seizures per week over the 12-week treatment period.

The median percent reduction in seizure frequency per week from baseline was $46 \%$ in the levetiracetam XR group and $33.4 \%$ in the placebo group. The estimated median percent reduction in seizures per week from baseline of levetiracetam XR over placebo was $14.4 \%$ (two-sided $95 \% \mathrm{CI}$ $0.9 \%-26.0 \% ; P=0.038)$ in the intention-to-treat population. The responder rate ( $\geq 50 \%$ reduction in seizure frequency per week from baseline) was $43 \%$ in the levetiracetam XR group and $29 \%$ in the placebo group. In addition, during the entire 12-week treatment period, 8/79 (10.1\%) of patients treated with levetiracetam XR were seizure-free in comparison to $1 / 79(1.3 \%)$ of placebo-treated patients.

\section{Pivotal trials for immediate release levetiracetam}

The initial approval of levetiracetam for the adjunctive treatment of partial-onset seizures in adults is based on the results of 3 double-blind, randomized, placebo-controlled, multicenter trials. ${ }^{2-4}$ The American trial by Cereghino et al looked at the efficacy and tolerability of $500 \mathrm{mg}$ bid and $1500 \mathrm{mg}$ bid of levetiracetam as add-on therapy in patients 16 to 70 years of age with partial-onset seizures refractory to at least 2 AEDs. ${ }^{3}$ The study consisted of a 12-week placebo baseline period, a 4 -week drug uptitration period,

Table 2 Recommended dosing adjustment in adults with impaired renal function ${ }^{22}$

\begin{tabular}{lll}
\hline $\begin{array}{l}\text { Degree of renal } \\
\text { impairment }\end{array}$ & $\begin{array}{l}\text { Creatinine clearance } \\
\left(\mathbf{m L} / \mathbf{m i n} / 1.73 \mathbf{~ m}^{2}\right)\end{array}$ & Dose per day \\
\hline Normal & $>80$ & 1000 to $3000 \mathrm{mg}$ \\
Mild & 50 to 80 & 1000 to $2000 \mathrm{mg}$ \\
Moderate & 30 to 50 & 500 to $1500 \mathrm{mg}$ \\
Severe & $<30$ & 500 to $1000 \mathrm{mg}$ \\
\hline
\end{tabular}

and a 14-week treatment/evaluation period. Two hundred ninety-four patients were randomized: 98 to levetiracetam $1000 \mathrm{mg} /$ day, 101 to levetiracetam $3000 \mathrm{mg}$ /day and 95 to placebo. The median percent reduction in seizure frequency from baseline was 32.5\% with levetiracetam $1000 \mathrm{mg} /$ day, $37.1 \%$ with levetiracetam $3000 \mathrm{mg} /$ day, and $6.8 \%$ with placebo $(P<0.001)$. The median percent reduction in seizure frequency over placebo was $20.9 \%$ with levetiracetam $1000 \mathrm{mg} /$ day $(P<0.001)$ and $27.7 \%$ with levetiracetam $3000 \mathrm{mg} /$ day $(P<0.001)$. The responder rate was $33 \%$ with levetiracetam $1000 \mathrm{mg} /$ day, 39.8\% with levetiracetam $3000 \mathrm{mg} /$ day, and $10.8 \%$ with placebo $(P<0.001)$. Eight patients in the levetiracetam $3000 \mathrm{mg} /$ day group were seizure-free during the entire 14-week evaluation period compared to none in the placebo group $(P=0.01)$.

In one European trial by Shorvon et al, the efficacy and tolerability of $500 \mathrm{mg}$ bid and $1000 \mathrm{mg}$ bid of levetiracetam as add-on therapy in patients 16 to 65 years of age with partial-onset seizures refractory to 1 to 2 AEDs was assessed. ${ }^{4}$ The study consisted of an 8-week baseline period, a 4-week uptitration period, and a 12-week treatment/evaluation period. Three hundred twenty-four patients were randomized: 112 in the placebo group, 106 in the levetiracetam $1000 \mathrm{mg}$ /day group, and 106 in the levetiracetam $2000 \mathrm{mg}$ /day group. The median percent reduction in weekly seizure frequency from baseline was $17.7 \%$ with levetiracetam $1000 \mathrm{mg} /$ day $(P<0.001), 26.5 \%$ with levetiracetam $2000 \mathrm{mg} /$ day $(P<0.001)$, and $6.1 \%$ with placebo. The median percent reduction in seizure frequency over placebo was $16.4 \%$ with levetiracetam $1000 \mathrm{mg} /$ day $(P=0.006)$ and $17.7 \%$ with levetiracetam $2000 \mathrm{mg} /$ day $(P=0.003)$. The responder rate was $22.8 \%$ with levetiracetam $1000 \mathrm{mg} /$ day $(P<0.02)$, $31.6 \%$ with levetiracetam $2000 \mathrm{mg} /$ day $(P<0.001)$, and $10.4 \%$ with placebo.

In another European trial by Ben-Menachem and Falter, the efficacy and tolerability of levetiracetam $3000 \mathrm{mg} /$ day as add-on therapy in patients 16 to 70 years of age with partial-onset seizures was assessed. ${ }^{2}$ The study consisted of a 12-week baseline period, a 4-week up-titration period, a 14-week add-on evaluation period, and a monotherapy phase (for those who responded well to levetiracetam or placebo) that included a maximum of 12 weeks of downtitration of concomitant AED and 12 weeks of monotherapy with levetiracetam $3000 \mathrm{mg} /$ day. The primary efficacy variable was the percentage of patients who completed the monotherapy phase. Two hundred eighty-six patients were randomized: 181 to levetiracetam $3000 \mathrm{mg}$ /day and 105 to placebo. Of this intention-to-treat population, $19.9 \%$ of 
patients in the levetiracetam group completed the study in comparison to $9.5 \%$ in the placebo group $(P=0.029)$. In reference to the add-on phase, the median percent reduction in seizure frequency from baseline was $39.9 \%$ with levetiracetam and $7.2 \%$ with placebo $(P<0.001)$. The responder rate was $42.1 \%$ with levetiracetam and $16.7 \%$ with placebo $(P<0.001)$. Fourteen patients in the levetiracetam group $(8.2 \%)$ remained seizure-free during the add-on evaluation period vs one in the placebo group $(P=0.012)$. The results of the monotherapy phase are discussed below.

\section{Pivotal trial of immediate release levetiracetam in children}

The efficacy of levetiracetam XR has not been studied in children. However, levetiracetam IR is FDA approved for the adjunctive treatment of refractory partial-onset seizures in children above the age of 4 based on a double-blind, placebocontrolled, multicenter trial involving an intention-to-treat population of 198 patients 4 to 16 years of age that demonstrated a $26.8 \%$ reduction in weekly seizure frequency from baseline in patients taking $60 \mathrm{mg} / \mathrm{kg} /$ day of levetiracetam vs placebo. ${ }^{34}$ The pattern and incidence of treatment-emergent adverse events were similar in the levetiracetam and placebo groups. Several smaller studies have supported the efficacy and safety of add-on levetiracetam in children with refractory partial-onset seizures. ${ }^{35-38}$

\section{Idiopathic generalized epilepsies}

Levetiracetam also appears to be effective in the treatment of idiopathic generalized epilepsies. One recent randomized, double-blind, placebo-controlled, multicenter trial of adjunctive treatment with levetiracetam IR $3000 \mathrm{mg} /$ day in 120 patients $\geq 12$ years of age with myoclonic seizures associated with idiopathic generalized epilepsies showed a reduction of $\geq 50 \%$ in the number of days/week with myoclonic seizures in $58.3 \%$ of patients in the levetiracetam group and in $23.3 \%$ of patients in the placebo group $(P<0.001)$. Freedom from myoclonic seizures was seen in $25 \%$ of patients in the levetiracetam group and $5 \%$ of patients in the placebo group $(P=0.004)$. Freedom from all seizure types occurred in $21.7 \%$ of levetiracetamtreated patients and in $1.7 \%$ of placebo-treated patients $(P<0.001){ }^{39}$

Another randomized, double-blind, placebo-controlled, multicenter, parallel-group study evaluated the efficacy and tolerability of adjunctive levetiracetam in 164 patients 4 to 65 years of age with uncontrolled generalized tonic-clonic (GTC) seizures associated with idiopathic generalized epilepsies. The mean reduction in GTC seizure frequency per week was $56.5 \%$ in the levetiracetam group and $28.2 \%$ in the placebo group $(P=0.004)$. The responder rate was $72.2 \%$ for the levetiracetam group and $45.2 \%$ for the placebo group $(P<0.001)$. The percent of patients free of all seizures was $24.1 \%$ for levetiracetam-treated patients and $8.3 \%$ for placebo-treated patients $(P=0.009) .{ }^{40}$

\section{Monotherapy and newly diagnosed epilepsy}

Levetiracetam XR has not been studied in monotherapy. As described earlier, one of the pivotal levetiracetam IR trials included a 12-week monotherapy phase with levetiracetam $3000 \mathrm{mg} / \mathrm{day}^{2}{ }^{2}$ Only patients who responded well to levetiracetam or placebo during the add-on phase were eligible to enter the monotherapy phase. Of the 239 patients who completed the add-on phase, 86 were eligible to enter the monotherapy phase (placebo, $\mathrm{n}=17$; levetiracetam, $n=69$ ). For ethical reasons, 8/17 placebotreated patients were switched to levetiracetam but remained in the placebo group for analyses. After meeting escape criteria, 25 patients were withdrawn during the downtitration period. Out of the 69 levetiracetam-treated patients, 49 were successfully downtitrated to monotherapy and 36 (19.9\%) completed the study. Of the 17 placebo-treated patients, 12 were successfully down-titrated to monotherapy and $10(9.5 \%)$ completed the study (4 of which had been switched to levetiracetam). The median percent reduction in seizure frequency from baseline during the monotherapy evaluation period was $73.8 \%(P=0.037)$. The responder rate was $59.2 \%$. Nine of the 49 patients taking levetiracetam were seizure-free. This study suggested that levetiracetam $3000 \mathrm{mg} /$ day may be effective as monotherapy in the treatment of patients with partial-onset seizures refractory to other AEDs; however, neither the FDA nor the American Academy of Neurology have endorsed this use due to the unusual trial design. ${ }^{41}$

A large multicenter, double-blind, noninferiority, parallel-group trial assessed the efficacy and tolerability of levetiracetam in comparison to controlled-release carbamazepine as first treatment in 579 patients with newly diagnosed epilepsy. This study found equivalent seizure freedom rates in both groups at 6 months (73\% levetiracetam; $72.8 \%$ controlled-release carbamazepine). There was a trend towards higher withdrawal rates due to adverse events in the carbamazepine group when compared to the levetiracetam group (19.2\% vs 14.4\%); however, this was not statistically significant. ${ }^{42}$ Moreover, several retrospective studies 
have suggested that levetiracetam is effective and safe as monotherapy in adults with epilepsy. ${ }^{43-46}$

\section{Safety and tolerability}

\section{Adverse events in pivotal adult trials}

In its pivotal trial, levetiracetam XR was generally well tolerated. ${ }^{1}$ Overall, the incidence of adverse events did not significantly differ between the placebo and treatment groups. Treatment-emergent adverse reactions that had a higher incidence with levetiracetam XR compared with placebo were somnolence, irritability, dizziness, nausea, influenza and nasopharyngitis (Table 3). Headache was reported more frequently in the placebo arm. Five patients in the levetiracetam group discontinued due to adverse events (mouth ulceration, skin rash, asthenia, seizures) that were considered by the investigators to be causally related to the medication. Two patients in the placebo arm discontinued treatment due to adverse events. There were no clinically relevant changes from baseline in vital signs, body weight, laboratory values, electrocardiograms or physical examination findings. ${ }^{1}$

Furthermore, in the original pivotal trials that led to FDA approval of levetiracetam, treatment-emergent adverse reactions reported more often with levetiracetam IR than placebo were somnolence, asthenia, dizziness, headache, infection, rhinitis, and flu syndrome. ${ }^{47}$ Infections consisted mostly of common colds and upper respiratory infections that were not associated with elevated white blood cell counts. ${ }^{48}$ Of note, the IR trials used a different coding dictionary (COSTART) than the XR study (Med-DRA).

\section{Long-term studies and tolerability}

The pivotal trials for levetiracetam XR and IR are short-term studies of efficacy and tolerability that do not assess longterm outcomes. Thus far, there are no studies investigating the long-term outcomes of patients treated with levetiracetam XR. There are several for the IR formulation.

Table 3 Adverse events reported by $\geq 5 \%$ of patients in the levetiracetam $X R$ group'

\begin{tabular}{lll}
\hline Adverse event & Levetiracetam XR & Placebo \\
\hline Somnolence & $7.8 \%$ & $2.5 \%$ \\
Influenza & $7.8 \%$ & $3.8 \%$ \\
Irritability & $6.5 \%$ & 0 \\
Nasopharyngitis & $6.5 \%$ & $5.1 \%$ \\
Nausea & $5.2 \%$ & $2.5 \%$ \\
Dizziness & $5.2 \%$ & $2.5 \%$ \\
\hline
\end{tabular}

In a 2-year study of 301 patients 1 to 75 years of age with refractory epilepsy on levetiracetam IR, $45.8 \%$ discontinued treatment during the 24 month follow-up period. Discontinuation of levetiracetam was due to an adverse event in $6 \%$ and due to a combination of lack of efficacy and adverse events in $16.3 \%$. The most frequently reported side effects at the time of discontinuation were tiredness (13.8\%), agitation, aggression and hyperirritability (13.8\%), depression and apathy $(13.1 \%)$, and sleepiness $(8.5 \%) .{ }^{49}$

In another large cohort of 811 patients with refractory epilepsy 14 to 79 years of age on levetiracetam IR, the 3 -year retention rate was $58 \%$. Discontinuation was due to an adverse event in $10 \%$ and due to a combination of adverse events and lack of efficacy in 9\%. The most frequent adverse events were somnolence, dizziness, headache, behavior problems, gastrointestinal disturbances and skin rash. ${ }^{50}$

Additionally, a follow-up study of 280 patients 17 to 72 years of age with refractory partial-onset seizures that benefited from levetiracetam IR during one of the original pivotal trials $^{3}$ was performed. Eighty-one patients (28.9\%) terminated the study prematurely. Of these, 39 (13.9\%) withdrew due to loss or lack of efficacy and $20(7.1 \%)$ withdrew due to adverse events. This included 5 patients who died (deaths considered by investigator to be unrelated to treatment) and 3 patients who withdrew due to a behavioral adverse event (aggression, irritability, intentional overdose)..$^{51}$

In a prospective analysis of 354 patients with refractory epilepsy on levetiracetam IR followed for 2 years, sedation was the most common side effect (10.7\%). However, mood disturbance, which occurred in 17 patients (4.8\%), was more likely to lead to discontinuation of treatment. This study also found that a slower titration regimen was associated with an increased rate of retention. ${ }^{52}$

\section{Psychiatric and behavioral adverse events}

Behavioral adverse events were noted in open-label studies and post-marketing analyses of levetiracetam IR. A systematic review of levetiracetam's safety profile reported the incidence of behavioral problems (agitation, anti-social reaction, anxiety, apathy, depersonalization, depression, emotional lability, euphoria, hostility, nervousness, neurosis, and personality disorder) in placebo-controlled epilepsy trials at $13.5 \%$ in the levetiracetam IR group vs $6 \%$ in the placebo group. ${ }^{48}$ Behavioral adverse events did not appear to be dose-related, and there was no correlation to whether the patient was a treatment responder. Therefore, the theory of "forced normalization" appears not to apply. Further logistic regression analyses also revealed that patients with a previous 
history of psychiatric disorders were more likely to report behavioral adverse events. ${ }^{47,48}$

In a study of 517 patients on levetiracetam IR, 10.1\% developed a psychiatric adverse effect (3.5\% aggressive behavior, $2.5 \%$ affective disorder, $2.3 \%$ emotional lability, $1.2 \%$ psychosis, and $0.6 \%$ other behavioral abnormalities). A significant association was found in patients with a history of status epilepticus, febrile convulsions, or previous psychiatric disorder. Concomitant use of lamotrigine appeared to have a protective effect. ${ }^{53}$

Interestingly, the incidence of affective symptoms is significantly higher among patients on levetiracetam for epilepsy than in those on it for cognition or anxiety, even when taken in similar doses. This raises the possibility that there is something inherently different in epilepsy patients that predisposes them to these behavioral side effects. ${ }^{54}$ Those with learning disabilities may also be at a higher risk. In a study of 184 adults with epilepsy on levetiracetam IR, 30\% of which had a learning disability, there were significantly more behavioral adverse effects ( $23 \%$ vs $10 \%$ ) in those with learning disabilities than those without. ${ }^{55}$

\section{Tolerability in the elderly}

The pivotal trial for levetiracetam XR did not contain a sufficient number of patients $\geq 65$ years of age to reliably ascertain tolerability in this subgroup. However, conclusions can be drawn from trials involving the IR formulation.

In a subset analysis of the KEEPER trial, the efficacy and tolerability of levetiracetam as add-on therapy for partialonset seizures was evaluated in 78 patients $\geq 65$ years of age. Somnolence was the most common reported adverse event, occurring in $16.7 \%$. Dizziness occurred in $9 \%$. Discontinuation of treatment due to an adverse event occurred in $19.2 \%$ of patients. ${ }^{56}$

Cramer et al compared treatment-emergent side effects in levetiracetam trials of young and elderly patients with anxiety and cognitive disorders and young patients with epilepsy. The only significant difference in reported adverse events between the young and elderly groups occurred in those with anxiety disorders, with headache and tremor occurring more frequently in the elderly patients. Overall, levetiracetam was well tolerated by all groups. ${ }^{57}$

\section{Pregnancy and lactation}

Levetiracetam XR falls under pregnancy category $\mathrm{C}$, to be used only if the potential benefit justifies the potential risk to the fetus. Isoherranen et al used a well established mouse model of AED teratogenicity to study the developmental outcomes of fetuses exposed to levetiracetam or its major metabolite in humans, 2-pyrrolidone $N$-butyric acid (PBA). They found significantly reduced fetal weights in most groups and an increased incidence of skeletal abnormalities in both the PBA and levetiracetam groups. Two out of 107 fetuses exposed to $1200 \mathrm{mg} / \mathrm{kg} /$ day of levetiracetam had cleft palate. Otherwise, no gross structural malformations among the exposed embryos were found, including those receiving a higher dose of levetiracetam $(2000 \mathrm{mg} / \mathrm{kg} /$ day $){ }^{58}$

In 2006, the UK Epilepsy and Pregnancy Register reported pregnancy outcomes in a cohort of women with epilepsy and first-trimester exposure to levetiracetam IR..$^{59}$ There were a total of 117 pregnancies, 39 of which were monotherapy exposures. Three of the 117 exposed pregnancies had a major congenital malformation. All of these occurred in pregnancies also exposed to other AEDs. The malformations included pyloric stenosis and spina bifida. Five minor congenital malformations occurred, also all in pregnancies exposed to polytherapy.

Additionally, in a study of 14 women with epilepsy on levetiracetam IR during pregnancy ( 8 on concomitant AEDs), breast milk levetiracetam concentrations and maternal, umbilical cord and infant levetiracetam plasma concentrations were studied. ${ }^{60}$ The mean milk: maternal plasma concentration of levetiracetam was 1.05, suggesting extensive transfer into breast milk. Substantial placental transfer of levetiracetam was also found, and the estimated elimination half-life of levetiracetam in 13 neonates was 18 hours. However, the plasma concentrations in the infants were, on average, $13 \%$ that of maternal plasma concentrations. In light of the significant transfer of levetiracetam from mother to fetus and into breast milk, the low levetiracetam concentrations in breast-fed neonates suggest rapid elimination of levetiracetam in neonates.

\section{Discussion}

Levetiracetam has many unique characteristics in regards to its mechanism of action: it lacks anticonvulsive activity in the maximal electric shock and the pentylenetetrazol seizure tests; it does not act at the three main sites of conventional AEDs; and it appears to offer diseasemodifying benefits in genetic and kindled epilepsy models. Thus far, only sodium valproate and, to a lesser extent, phenobarbital have demonstrated antiepileptogenic properties in the kindling model, ${ }^{61}$ but only do so at doses large enough to cause significant sedation and ataxia. Neither drug demonstrates antiepileptic properties in the genetic 
spontaneously epileptic rat (SER) model. ${ }^{11}$ All of these qualities distinguish levetiracetam from other first and second-generation AEDs.

Whether the antiepileptic properties of levetiracetam in kindled models translate to the clinical arena is uncertain when consideration is given to the fact that clinical epilepsy, with the exception of a few rare syndromes, is not a progressive, self-perpetuating disorder. ${ }^{62}$ Levetiracetam has only demonstrated a delay in the development or an increase in the threshold required to acquire epilepsy in the models reviewed by this article, suggesting that it is a disease-modifying agent rather than being truly antiepileptogenic. ${ }^{63}$ Furthermore, the protection levetiracetam afforded in the genetic SER model may not hold up in clinical practice considering the rats have specific mutations which have not been correlated in human causes of epilepsy and may make them metabolically and pharmacologically different.

Interestingly, levetiracetam appears to target sites previously not involved in anticonvulsive therapy, including high-voltage calcium channels and the SV2A receptor. These new mechanisms of action may contribute to levetiracetam's low incidence of drug-drug interactions, making it especially useful in cases of refractory epilepsy in which polypharmacy often leads to increased adverse events.

An ideal antiepileptic drug is one that is efficacious in controlling seizures with no adverse events. The pharmacokinetic characteristics of this ideal AED would include: rapid absorption, good bioavailability, rapid achievement of steady-state concentrations, linear kinetics, minimal protein binding, absence of significant drug-drug interactions, a halflife allowing daily or bid dosing, a wide therapeutic index, and no metabolism. ${ }^{24}$

Levetiracetam XR exhibits many of these ideal characteristics. It is rapidly and almost completely absorbed after oral ingestion, is $<10 \%$ protein-bound, demonstrates linear kinetics, is minimally metabolized via a pathway independent of the cytochrome P450 system, has no active metabolites, has no significant drug-drug interactions, allows for oncedaily dosing, and has a wide therapeutic index. Levetiracetam $\mathrm{XR}$ is ideal for patients with multiple medical problems on polytherapy and those with hepatic impairment. In addition, preliminary data suggest that levetiracetam is a good AED option for patients on chemotherapeutic agents. ${ }^{64,65}$ However, given that levetiracetam is renally excreted, dose adjustments are needed in patients with renal impairment and in some elderly patients due to an age-related decrease in creatinine clearance.
The efficacy, safety, and tolerability of levetiracetam for the adjunctive treatment of partial-onset seizures have been clearly proven. Additional studies have suggested efficacy of levetiracetam IR in the treatment of idiopathic generalized epilepsies and as monotherapy. Levetiracetam XR was well-tolerated in its pivotal trial. The most common adverse events reported were somnolence, irritability, dizziness, nausea, influenza, and nasopharyngitis. In addition, levetiracetam does not alter body weight, ${ }^{66}$ which may be a favorable characteristic. The most concerning associated adverse events are behavioral in nature and include agitation, anxiety, depression, nervousness, irritability and, rarely, psychosis. Those with learning disabilities and with a previous history of psychiatric illness may be at higher risk. Behavioral adverse events are reversible with discontinuation of levetiracetam. ${ }^{41}$

Data on pregnancy outcomes are limited; however, the UK Epilepsy and Pregnancy Register's report on 117 pregnancies exposed to levetiracetam IR showed promising outcomes with only 3 major congenital malformations, all in polytherapy exposures. However, data on levetiracetam's possible effect on cognitive development are lacking.

One of the possible benefits of levetiracetam XR is its once-daily dosing schedule because once and twicedaily dosing have been shown to result in a higher rate of compliance, and there appears to be an inverse relationship between the number of daily medication doses and compliance. ${ }^{67,68}$ Furthermore, because of relatively constant plasma concentrations, extended release formulations may minimize concentration-related adverse effects. These benefits should be weighed against the potential higher risk of a breakthrough seizure if a dose of a once-daily medication is missed, as the impact of a missed dose is greater the larger the dose and the less frequent the administration schedule. ${ }^{69}$

\section{Conclusion}

Extended release levetiracetam is a truly unique and valuable antiepileptic drug that can be added to the arsenal of existing treatment options for patients with epilepsy. Its favorable pharmacokinetic properties, lack of significant drug-drug interactions, overall safety and tolerability, and convenient once-daily dosing make levetiracetam XR an ideal add-on therapy for the treatment of partial-onset seizures. Future research will undoubtedly shed more light on the role of the SV2A receptor in the pathophysiology of epilepsy and possibly other hereto undiscovered biopharmacologic properties of levetiracetam. 


\section{Disclosure}

The author discloses no conflicts of interest.

\section{References}

1. Peltola J, Coetzee C, Jiménez F, et al. Once-daily extended-release levetiracetam as adjunctive treatment of partial-onset seizures in patients with epilepsy: a double-blind, randomized, placebo-controlled trial. Epilepsia. 2009;50(3):406-414.

2. Ben-Menachem E, Falter U. Efficacy and tolerability of levetiracetam $3000 \mathrm{mg} / \mathrm{d}$ in patients with refractory partial seizures: a multicenter, double-blind, responder-selected study evaluating monotherapy. European Levetiracetam Study Group. Epilepsia. 2000;41(10):1276-1283.

3. Cereghino JJ, Biton V, Abou-Khalil B, Dreifuss F, Gauer LJ, Leppik I. Levetiracetam for partial seizures: results of a double-blind, randomized clinical trial. Neurology. 2000;55(2):236-242.

4. Shorvon SD, Löwenthal A, Janz D, Bielen E, Loiseau P. Multicenter double-blind, randomized, placebo-controlled trial of levetiracetam as add-on therapy in patients with refractory partial seizures. European Levetiracetam Study Group. Epilepsia. 2000;41(9):1179-1186.

5. Rouits E, Burton I, Guénolé E, Troenaru MM, Stockis A, SargentiniMaier ML. Pharmacokinetics of levetiracetam XR $500 \mathrm{mg}$ tablets. Epilepsy Res. 2009;84(2-3):224-231.

6. Noyer M, Gillard M, Matagne A, Hénichart JP, Wülfert E. The novel antiepileptic drug levetiracetam (ucb L059) appears to act via a specific binding site in CNS membranes. Eur J Pharmacol. 1995;286(2):137-146.

7. Klitgaard H, Matagne A, Gobert J, Wülfert E. Evidence for a unique profile of levetiracetam in rodent models of seizures and epilepsy. Eur J Pharmacol. 1998;353(2-3):191-206.

8. Goddard GV, McIntyre DC, Leech CK. A permanent change in brain function resulting from daily electrical stimulation. Exp Neurol. 1969;25(3):295-330.

9. Vinogradova LV, van Rijn CM. Anticonvulsive and antiepileptogenic effects of levetiracetam in the audiogenic kindling model. Epilepsia. 2008;49(7):1160-1168.

10. Löscher W, Hönack D, Rundfeldt C. Antiepileptogenic effects of the novel anticonvulsant levetiracetam (ucb L059) in the kindling model of temporal lobe epilepsy. J Pharmacol Exp Ther. 1998;284(2):474-479.

11. Ji-qun C, Ishihara K, Nagayama T, Serikawa T, Sasa M. Long-lasting antiepileptic effects of levetiracetam against epileptic seizures in the spontaneously epileptic rat (SER): differentiation of levetiracetam from conventional antiepileptic drugs. Epilepsia. 2005;46(9):1362-1370.

12. Zona C, Niespodziany I, Marchetti C, Klitgaard H, Bernardi G, Margineanu DG. Levetiracetam does not modulate neuronal voltagegated Na+ and T-type Ca2+ currents. Seizure. 2001;10(4):279-286.

13. Lukyanetz EA, Shkryl VM, Kostyuk PG. Selective blockade of N-type calcium channels by levetiracetam. Epilepsia. 2002;43(1):9-18.

14. Niespodziany I, Klitgaard H, Margineanu DG. Levetiracetam inhibits the high-voltage-activated $\mathrm{Ca}(2+)$ current in pyramidal neurones of rat hippocampal slices. Neurosci Lett. 2001;306(1-2):5-8.

15. Angehagen M, Margineanu DG, Ben-Menachem E, Rönnbäck L, Hansson E, Klitgaard H. Levetiracetam reduces caffeine-induced $\mathrm{Ca} 2+$ transients and epileptiform potentials in hippocampal neurons. Neuroreport. 2003;14(3):471-475.

16. Nagarkatti N, Deshpande LS, DeLorenzo RJ. Levetiracetam inhibits both ryanodine and IP3 receptor activated calcium induced calcium release in hippocampal neurons in culture. Neurosci Lett. 2008;436(3):289-293.

17. Desaulles E, Boux O, Feltz P. Caffeine-induced $\mathrm{Ca} 2+$ release inhibits GABAA responsiveness in rat identified native primary afferents. Eur J Pharmacol. 1991;203(1):137-140.

18. Margineanu DG, Klitgaard H. Inhibition of neuronal hypersynchrony in vitro differentiates levetiracetam from classical antiepileptic drugs. Pharmacol Res. 2000;42(4):281-285.

19. Lynch BA, Lambeng N, Nocka K, et al. The synaptic vesicle protein SV2A is the binding site for the antiepileptic drug levetiracetam. Proc Natl Acad Sci U S A. 2004;101(26):9861-9866.
20. Gillard M, Chatelain P, Fuks B. Binding characteristics of levetiracetam to synaptic vesicle protein 2A (SV2A) in human brain and in $\mathrm{CHO}$ cells expressing the human recombinant protein. Eur J Pharmacol. 2006;536(1-2):102-108.

21. Crowder KM, Gunther JM, Jones TA, et al. Abnormal neurotransmission in mice lacking synaptic vesicle protein 2A (SV2A). Proc Natl Acad Sci U S A. 1999;96(26):15268-15273.

22. Keppra XR [Package insert]. Smyrna, GA: UCB Pharma, Inc, 2008.

23. Patsalos PN. Clinical pharmacokinetics of levetiracetam. Clin Pharmacokinet. 2004;43(11):707-724.

24. Patsalos PN. Pharmacokinetic profile of levetiracetam: toward ideal characteristics. Pharmacol Ther. 2000;85(2):77-85.

25. Brockmöller J, Thomsen T, Wittstock M, Coupez R, Lochs H, Roots I. Pharmacokinetics of levetiracetam in patients with moderate to severe liver cirrhosis (Child-Pugh classes A, B, and C): characterization by dynamic liver function tests. Clin Pharmacol Ther. 2005;77(6):529-541.

26. Glauser TA, Mitchell WG, Weinstock A, et al. Pharmacokinetics of levetiracetam in infants and young children with epilepsy. Epilepsia. 2007;48(6):1117-1122.

27. Pellock JM, Glauser TA, Bebin EM, et al. Pharmacokinetic study of levetiracetam in children. Epilepsia. 2001;42(12):1574-1579.

28. Browne TR, Szabo GK, Leppik IE, Josephs E, Paz J, Baltes E, Jensen CM. Absence of pharmacokinetic drug interaction of levetiracetam with phenytoin in patients with epilepsy determined by new technique. J Clin Pharmacol. 2000;40(6):590-595.

29. Ragueneau-Majlessi I, Levy RH, Meyerhoff C. Lack of effect of repeated administration of levetiracetam on the pharmacodynamic and pharmacokinetic profiles of warfarin. Epilepsy Res. 2001;47(1-2): 55-63.

30. Levy RH, Ragueneau-Majlessi I, Baltes E. Repeated administration of the novel antiepileptic agent levetiracetam does not alter digoxin pharmacokinetics and pharmacodynamics in healthy volunteers. Epilepsy Res. 2001;46(2):93-99.

31. Ragueneau-Majlessi I, Levy RH, Janik F. Levetiracetam does not alter the pharmacokinetics of an oral contraceptive in healthy women. Epilepsia. 2002;43(7):697:702.

32. Gidal BE, Baltès E, Otoul C, Perucca E. Effect of levetiracetam on the pharmacokinetics of adjunctive antiepileptic drugs: a pooled analysis of data from randomized clinical trials. Epilepsy Res. 2005; 64(1-2):1-11.

33. Perucca E, Gidal BE, Baltès E. Effects of antiepileptic comedication on levetiracetam pharmacokinetics: a pooled analysis of data from randomized adjunctive therapy trials. Epilepsy Res. 2003;53(1-2):47-56.

34. Glauser TA, Ayala R, Elterman RD, et al. Double-blind placebocontrolled trial of adjunctive levetiracetam in pediatric partial seizures. Neurology. 2006;66(11):1654-1660.

35. Glauser TA, Pellock JM, Bebin EM, et al. Efficacy and safety of levetiracetam in children with partial seizures: an open-label trial. Epilepsia. 2002;43(5):518-524.

36. Grosso S, Franzoni E, Coppola G, et al. Efficacy and safety of levetiracetam: an add-on trial in children with refractory epilepsy. Seizure. 2005;14(4):248-253.

37. Lagae L, Buyse G, Ceulemans B. Clinical experience with levetiracetam in childhood epilepsy: an add-on and mono-therapy trial. Seizure. 2005;14(1):66-71.

38. Callenbach PM, Arts WF, ten Houten R, et al. Add-on levetiracetam in children and adolescents with refractory epilepsy: results of an open-label multi-centre study. Eur J Paediatr Neurol. 2008;12(4): 321-327.

39. Noachtar S, Andermann E, Meyvisch P, et al. Levetiracetam for the treatment of idiopathic generalized epilepsy with myoclonic seizures. Neurology. 2008;70(8):607-616.

40. Berkovic SF, Knowlton RC, Leroy RF, Schiemann J, Falter U; Levetiracetam N01057 Study Group. Placebo-controlled study of levetiracetam in idiopathic generalized epilepsy. Neurology. 2007;69(18):1751-1760.

41. Safdieh J, Harden C. Levetiracetam: past, present and future. Future Medicine. 2006;1(4):365-371. 
42. Brodie MJ, Perucca E, Ryvlin P, Ben-Menachem E, Meencke HJ; Levetiracetam Monotherapy Study Group. Comparison of levetiracetam and controlled-release carbamazepine in newly diagnosed epilepsy. Neurology. 2007;68(6):402-408.

43. Alsaadi TM, Shatzel A, Marquez AV, Jorgensen J, Farias S. Clinical experience of levetiracetam monotherapy for adults with epilepsy: 1-year follow-up study. Seizure. 2005;14(2):139-142.

44. Alsaadi TM, Koopmans S, Apperson M, Farias S. Levetiracetam monotherapy for elderly patients with epilepsy. Seizure. 2004;13(1):58-60.

45. Alsaadi TM, Thieman C. Levetiracetam monotherapy for newly diagnosed epilepsy patients. Seizure. 2003;12(3):154-156.

46. Alsaadi TM, Thieman C, Zusman EE. Levetiracetam monotherapy for adults with localization-related epilepsy. Epilepsy Behav. 2002;3(5):471-474.

47. Abou-Khalil B. Benefit-risk assessment of levetiracetam in the treatment of partial seizures. Drug Saf. 2005;28(10):871-890.

48. French J, Edrich P, Cramer JA. A systematic review of the safety profile of levetiracetam: a new antiepileptic drug. Epilepsy Res. 2001;47(1-2):77-90.

49. Bootsma HP, Ricker L, Diepman L, et al. Levetiracetam in clinical practice: long-term experience in patients with refractory epilepsy referred to a tertiary epilepsy center. Epilepsy Behav. 2007;10(2):296-303.

50. Depondt C, Yuen AW, Bell GS, et al. The long term retention of levetiracetam in a large cohort of patients with epilepsy. J Neurol Neurosurg Psychiatry. 2006;77(1):101-103.

51. Abou-Khalil B, Schaich L. Long-term efficacy of levetiracetam for partial seizures. Seizure. 2005;14(8):577-585.

52. Nicolson A, Lewis SA, Smith DF. A prospective analysis of the outcome of levetiracetam in clinical practice. Neurology. 2004;63(3):568-570.

53. Mula M, Trimble MR, Yuen A, Liu RS, Sander JW. Psychiatric adverse events during levetiracetam therapy. Neurology. 2003;61(5): 704-706.

54. Cramer JA, De Rue K, Devinsky O, Edrich P, Trimble MR. A systematic review of the behavioral effects of levetiracetam in adults with epilepsy, cognitive disorders, or an anxiety disorder during clinical trials. Epilepsy Behav. 2003;4(2):124-132.

55. Brodtkorb E, Klees TM, Nakken KO, Lossius R, Johannessen SI. Levetiracetam in adult patients with and without learning disability: focus on behavioral adverse effects. Epilepsy Behav. 2004;5(2):231-235.

56. Ferrendelli JA, French J, Leppik I, et al. Use of levetiracetam in a population of patients aged 65 years and older: a subset analysis of the KEEPER trial. Epilepsy Behav. 2003;4(6):702-709.
57. Cramer JA, Leppik IE, Rue KD, Edrich P, Krämer G. Tolerability of levetiracetam in elderly patients with CNS disorders. Epilepsy Res. 2003;56(2-3):135-145.

58. Isoherranen N, Spiegelstein O, Bialer M, et al. Developmental outcome of levetiracetam, its major metabolite in humans, 2-pyrrolidinone N-butyric acid, and its enantiomer (R)-alpha-ethyloxo-pyrrolidine acetamide in a mouse model of teratogenicity. Epilepsia. 2003;44(10):1280-1288.

59. Hunt S, Craig J, Russell A, et al. Levetiracetam in pregnancy: preliminary experience from the UK Epilepsy and Pregnancy Register. Neurology. 2006;67(10):1876-1879.

60. Tomson T, Palm R, Källén K, et al. Pharmacokinetics of levetiracetam during pregnancy, delivery, in the neonatal period, and lactation. Epilepsia. 2007;48(6):1111-1116.

61. Silver JM, Shin C, McNamara JO. Antiepileptogenic effects of conventional anticonvulsants in the kindling model of epilespy. Ann Neurol. 1991;29(4):356-363.

62. Sills GJ. Seizures beget seizures: a lack of experimental evidence and clinical relevance fails to dampen enthusiasm. Epilepsy Curr. 2007;7(4):103-104.

63. Dudek FE, Bertram EH, Staley KJ. Antiepileptogenesis therapy with levetiracetam: data from kindling versus status epilepticus models. Epilepsy Curr. 2008;8(1):28-30.

64. Lim DA, Tarapore P, Chang E, et al. Safety and feasibility of switching from phenytoin to levetiracetam monotherapy for glioma-related seizure control following craniotomy: a randomized phase II pilot study. J Neurooncol. 2009 Jan 24. Epub ahead of print.

65. Wagner GL, Wilms EB, Van Donselaar CA, Vecht ChJ. Levetiracetam: preliminary experience in patients with primary brain tumours. Seizure. 2003;12(8):585-586.

66. Gidal BE, Sheth RD, Magnus L, Herbeuval AF. Levetiracetam does not alter body weight: analysis of randomized, controlled clinical trials. Epilepsy Res. 2003;56(2-3):121-126.

67. Claxton AJ, Cramer J, Pierce C. A systematic review of the associations between dose regimens and medication compliance. Clin Ther. 2001;23(8):1296-1310.

68. Pullar T, Birtwell AJ, Wiles PG, Hay A, Feely MP. Use of a pharmacologic indicator to compare compliance with tablets prescribed to be taken once, twice, or three times daily. Clin Pharmacol Ther. 1988;44(5):540-545.

69. Bialer M. Extended-release formulations for the treatment of epilepsy. CNS Drugs. 2007;21(9):765-774.
Neuropsychiatric Disease and Treatment

\section{Publish your work in this journal}

Neuropsychiatric Disease and Treatment is an international, peerreviewed journal of clinical therapeutics and pharmacology focusing on concise rapid reporting of clinical or pre-clinical studies on a range of neuropsychiatric and neurological disorders. This journal is indexed on PubMed Central, the 'PsycINFO' database and CAS, and is the official

\section{Dovepress}

journal of The International Neuropsychiatric Association (INA). The manuscript management system is completely online and includes a very quick and fair peer-review system, which is all easy to use. Visit http://www.dovepress.com/testimonials.php to read real quotes from published authors. 